\title{
Manipulations to the timing and type of instructions to examine motor skill performance under pressure
}

\section{Nicole T. Ong, Alison Bowcock and Nicola J. Hodges*}

School of Human Kinetics, The University of British Columbia, Vancouver, BC, Canada

\section{Edited by:}

David L. Wright, Texas A\&M University, USA

\section{Reviewed by:}

Suzete Chiviacowsky, Federal University of Pelotas, Brazil

Rob Gray, Arizona State University, USA

\section{*Correspondence:}

Nicola J. Hodges, School of Human Kinetics, The University of British Columbia, 210-6081 University Blvd, Vancouver, BC, Canada V6T 1Z1. e-mail: nicola.hodges@ubc.ca
There is evidence that prescriptive versus discovery methods of learning can lead to breakdowns under pressure due to "reinvestment" of knowledge and a more conscious, controlled mode of control. There is some speculation that this breakdown is mediated by the attentional focus of the instructions. We expected these effects to also be moderated by when in practice these instructions are given. Across two experiments, five groups practiced a forehand disk throwing task and we manipulated the timing and attentional focus of instructions. Internally directed instructions provided to participants early in practice resulted in a slower rate of acquisition (outcome error) and detrimental effects under stress, in comparison to the same instructions provided later in practice or not at all. Externally directed, technical instructions positively impacted rate of acquisition and regardless of when in practice they were provided, there were no adverse effects associated with instructions under pressure. These results show that the direction of attention encouraged by instructions moderates performance under stress as does the timing of presentation of these instructions.

Keywords: practice, motor learning, attentional focus, stress, choking

\section{GENERAL INTRODUCTION}

When stakes are high, even elite athletes are susceptible to cracking or "choking" under pressure. The phenomenon of choking has most recently been explained by the skill-focused attention theory (also referred to as the explicit monitoring hypothesis, Beilock and Carr, 2001; Gray, 2004) and the reinvestment hypothesis (also referred to as the consciousness processing hypothesis, Masters, 1992; Liao and Masters, 2002). Although these two theories share commonalities with respect to the type of mechanisms assumed to underlie performance decrements under stress, there are discrepant predictions with respect to the type of practice that will minimize the potential for choking. Little is known with regards to when in practice instructions should be optimally introduced and how this interacts with the attentional focus encouraged by these instructions, that is either internal or external (see Wulf et al., 1998; Wulf and Prinz, 2001; Wulf, 2007a,b). In the following experiments, we manipulate the timing of instructions during the acquisition of a novel, throwing skill, to determine its impact on retention and performance under pressure. Internally focused instructions given on either the first or second day of practice are compared in Experiment 1 and externally focused instructions are compared in Experiment 2.

The learning of motor skills is thought by many to progress through some typical phases as one advances from a beginner/novice level to a more skilled level or expert performance (Fitts, 1964; Fitts and Posner, 1967; Schneider and Shiffrin, 1977; Anderson, 1982, 1983). On the novice end, motor skill performance is thought to be guided by a step-by-step, conscious processing, of explicit, declarative knowledge. Working memory and attentional resources are thought to be heavily involved during this early stage of learning (Fitts and Posner, 1967). As the learner becomes more skilled in performance, the execution of the motor skill becomes more "automatic," that is, it seems to require little attention or cognitive effort. By this stage, the learner is thought to be guided by proceduralized or implicit knowledge that is not immediately accessible to consciousness (Fitts and Posner, 1967; Schneider and Shiffrin, 1977; Beilock and Carr, 2001, 2004).

Although well learned motor skills are believed to be controlled in a somewhat "automatic" manner, it has been proposed that under pressure, the performer can revert to a declarative, step-by-step conscious control of movement. This heightened attention is thought to adversely impact performance (Kimble and Perlmuter, 1970; Masters, 1992), and has been referred to as "choking" (Baumeister, 1984; Baumeister and Steinhilber, 1984; Lewis and Linder, 1997; Beilock and Carr, 2001). Choking is expected to affect skilled performers but not novices, who have been shown to benefit from the step-by-step, online monitoring of performance (Beilock et al., 2002; Gray, 2004) or at least not show adverse effects (Ford et al., 2005). This difference in performance as a function of skill level has become known as the skill-focused attention theory (or explicit monitoring hypothesis), whereby attention onto previously automatic processes involved with performance of the skill, interferes with skill execution (Beilock and Carr, 2001). One explanation for the regression in performance is a "dechunking" of previously integrated units into independently executed units of movement, which would increase the likelihood of variability and error in performance (Masters, 1992; Masters et al., 1993).

Similar ideas with respect to regressions in performance as a function of attention or knowledge have been forwarded by Masters (1992, see also Masters and Maxwell, 2004, 2008 for reviews). According to his "reinvestment hypothesis," under pressure, a performer would tend to control his/her performance of a motor skill in a conscious manner, using accumulated explicit (declarative) knowledge. In a number of experiments, Masters and colleagues found that as performers accumulated or reported more explicit 
knowledge (or verbal rules), the greater was their propensity to reinvest and show decrements under pressure (Masters and Maxwell, 2004, 2008; Maxwell et al., 2006).

While the skill-focused attention theory and reinvestment hypothesis are similar in their explanation of the mechanisms leading to choking, different predictions result as a function of the type of practice conditions which would best inoculate against performance decrements (i.e., choking; Baumeister, 1984; Beilock and Carr, 2001; Liao and Masters, 2002; Maxwell et al., 2006). According to predictions of the skill-focused attention theory, pressure-induced regressions in performance are only seen among participants who usually perform in a more automatic, less consciously controlled fashion, typically experts. Therefore, if a performer is encouraged to perform in a more consciously controlled fashion during practice, pressure-induced regressions in performance would not be expected. In support of this prediction, Beilock and Carr (2001) showed that when practice conditions (and processes) matched the performance conditions likely to be experienced under pressure, more skilled performers were able to avoid choking. That is, when performers adopted a more conscious, explicit mode of practicing, encouraged through the use of videotaping, they were better adapted to conditions prompted by anxiety and pressure such that performance levels were maintained under pressure. In contrast, an explicit, conscious mode of practice would be expected to harm later performance under pressure, as predicted by the reinvestment hypothesis. Accordingly, the more explicit knowledge or rules a learner acquires during practice, the greater the propensity for reinvestment and hence choking under pressure. Therefore Masters (1992) and Masters and Maxwell (2004) have recommended techniques to limit the build-up of explicit knowledge during practice, such as introducing secondary tasks which load on working memory.

An equally important consideration with respect to the type of practice conditions which are most likely to minimize performance decrements under pressure concerns the type of instructions provided. Although both Beilock and Carr (2001) and Masters (1992) discuss instructions with respect to skill-related knowledge and how an action should be performed (i.e., technical instruction), it has been shown that the locus of attention encouraged by these how-to instructions also moderates performance (Hodges and Franks, 2000; Wulf et al., 2002; Castaneda and Gray, 2007). Related to the prevention of choking is the idea that an external focus of attention promotes the automatic processing and execution of skills. While an external focus of attention directs a performer to the environmental or external effects of one's movements, an internal focus directs a performer's attention to his/her own body movements. This has become known as the constrained action hypothesis (Wulf et al., 2001; McNevin and Wulf, 2002). Evidence has been provided showing that an external focus of attention benefits both the performance and retention of motor skills, in comparison to a body-focused, internal attentional focus (see Wulf et al., 1998; Wulf, 2007a,b for reviews). Similar to the processes believed to underlie choking under pressure, it is believed that an internal focus encourages conscious control of movements and that when performers consciously control their movements, their efficiency is disrupted. This disruption is irrespective of the stage of learning; novice learner or more skilled performer, and the performance conditions; either high or low anxiety. Indeed, there is evidence that external attention benefits as a function of practice extend to transfer conditions where speed pressure has been induced in addition to attentional demanding conditions (Totsika and Wulf, 2003). Therefore, if external focus conditions encourage a more automatic type of control strategy, even when instructions or external cues are no longer provided, participants who practice with externally focused instructions would not be expected to be affected by stressinducing conditions to the same degree as participants who practice with internally focused instructions. The degree of self-focused attention, the mechanism implicated in performance breakdowns under pressure, is expected to be significantly more under internal versus external attention conditions.

In the following experiments we attempted to determine the optimal conditions for practice and performance under pressure, with respect to when instructions should be provided and the direction of attention encouraged by these instructions. In the first experiment, two groups of naïve participants were provided internal-focused instructions for a forehand Frisbee throw, during either the first or second session of two practice sessions. Both groups received the same amount of instruction, but differed in terms of when in practice this was received. They were compared to a third (control) group who did not receive instructions. In a second experiment we tested two further groups and again manipulated when in practice instructions were given. This time the instructions were changed to be externally focused. Our interest was not so much in comparing the impact of internally and externally directed instructions on retention, due to the large body of literature on this topic. Rather, we were concerned with how the timing of instruction affects performance under pressure, in order to test between the reinvestment hypothesis and the skill-focused attention hypothesis of choking. Because the direction of attention encouraged by these instructions was expected to modify the processes engaged during practice, these two attentional focus settings were tested across two experiments. We expected stronger effects in Experiment 1 with internally focused instructions due to the conscious processing assumed to be promoted by these instructions in comparison to externally focused instructions. According to the reinvestment hypothesis, if the amount of instruction and hence explicit knowledge or rules is an important predictor of performance under stress, then the two instruction groups relative to the control, irrespective of the attentional focus encouraged by the instructions, should show decrements under pressure. Further, we predicted that participants receiving instructions on the second day of practice (i.e., later in practice and closer to the retention/stress test) would be more likely to reinvest or choke under pressure due to the recency or saliency of this explicit, declarative knowledge and hence the accessibility of this knowledge under pressure (Masters, 1992).

If the skill-focused attention theory provides a better explanation for performance regressions under pressure, receiving instructions on the second day of practice would better inoculate performers against pressure inducing situations, due to the explicit, more conscious mode of performance that these instructions are expected to encourage. Hence the similarity in recent practice and assessment condition, whereby both engage declarative and conscious control, should benefit the second day instruction group. This recency effect of providing instructions on the second day was expected 
to be moderated by the type of attentional focus encouraged by the instructions. Because a more conscious mode of processing is expected to be encouraged by internally focused instructions, these timing related effects were only expected in Experiment 1 when the instructions were internally focused.

\section{EXPERIMENT 1 \\ INTRODUCTION}

Internally focused instructions concerning how to perform a forehand Frisbee throw were provided either on the first day of practice, on the second day of practice or not at all during practice. Participants who received instructions were expected to show decrements in performance under stress. If both amount of explicit knowledge accumulated and saliency or accessibility of these instructions (predictions of the reinvestment hypothesis) moderate performance under pressure, then the group receiving (internally focused) instructions on the second day was expected to show greater performance decrements than the group receiving the same instructions on the first day. If however, providing instructions on the second day prevents a more automatic mode of control and serves to inoculate performers against pressure (a prediction of the skill-focused attention theory), then only the group receiving internally focused instructions on the first day was expected to show performance decrements under pressure.

\section{METHOD}

\section{Participants}

Self-reported right-hand dominant participants (age range: 19-34 years) were recruited at the University of British Columbia and the neighborhoods close to its vicinity. Informed consent was obtained according to the ethical guidelines of the University of British Columbia (Behavioral Research Ethics Board) and participants filled out an experience questionnaire to confirm that they had no or only recreational Frisbee playing experience. A pretest was conducted consisting of five Frisbee forehand throws with the right hand. In addition to providing baseline performance measures, this enabled exclusion of participants who were able to perform the task well without practice. If a participant hit the target (as detailed below) three out of five times or hit the target two out of five times with unwavering straight flight paths of the Frisbee disk, they were excluded from the study $(n=6)$. Participants who passed the pretest criterion were quasi-randomly assigned to one of three groups with the constraint that the three groups were approximately matched in terms of their average absolute error values for the pretest: Internal Day 1 (INTD $1 ; n=10$; nine females; mean age $=21$ years), Internal Day 2 (INTD2; $n=10$; nine females; mean age $=24$ years), and Control (Ctrl; $n=9$; nine females; mean age $=21$ years) group.

\section{Task goals, materials, and apparatus}

The goal of the task was to throw a Frisbee flying-disk (Discraft 175 Ultra-Star ${ }^{\mathrm{TM}}$ flying-disc, $175 \mathrm{~g}$ ), with a straight flight path, to a target indicated as a red "X," located $100 \mathrm{~cm}$ from the ground and in the middle of a vertically positioned target board, $600 \mathrm{~cm}$ $(\mathrm{W}) \times 300 \mathrm{~cm}(\mathrm{H})$, marked every $25 \mathrm{~cm}$. A schematic diagram showing the setup and target grid is shown in Figure 1. Participants were to do this using a forehand throw and each throw was to be made from within a starting box positioned $50 \mathrm{~cm}$ behind a carpeted surface, $600 \mathrm{~cm}(\mathrm{~W}) \times 650 \mathrm{~cm}(\mathrm{~L})$, that lay between the starting box and the upright target board. The carpet was divided into $25 \mathrm{~cm} \times 25 \mathrm{~cm}$ grids, and a ruler was used to determine error to the nearest $5 \mathrm{~cm}$.

A forehand throw starts at the side of the body and is characterized by a forward motion of the arm. Typically, only two fingers are under the disk (in a V shape) and the disk is kept parallel to the ground during the throw. The wrist is pulled back and the elbow leads the throw. This skill was chosen because of its novelty and relative difficulty and supposed importance of technical instruction for novice throwers.

During the experiment, participants were reminded to pay attention to instructions, to remember and follow them closely (where appropriate) and aim to improve their performance on every trial. Eight internally focused instructions which comprised of 12 main body and position related cues were determined by an Ultimate Frisbee expert (past captain of the Canadian women's Ultimate team) before testing (see Table 1, left column). These instructions were further checked and validated with coaching-oriented websites. These instructions were provided both verbally and in written form to participants in both instruction groups on either one of the two testing days. A heart rate monitor (Polar Electro Oy, S810, China) was strapped to the participants' chest during each trial to provide measures of heart rate during the various experimental conditions. Light-emitting diodes (LEDs) were also attached to five sites on the right side of participants' body; the proximal end of the first metacarpal, radial tuberosity, medial and lateral condyles of humerus, and acromial process on the shoulder. Two video cameras (Canon NTSC Elura 70 Digital, Japan) recorded the movement form and outcome of the throw for all trials. The LEDs helped the experimenters analyze movement form from videos using Dartfish software (Dartfish, USA). During the pressure test the movement form video camera was connected to a projector (Optoma EP 738, Taiwan), enabling life-size video projection of the participant between stress-inducing trials. Images were projected onto a large Cinefold (IN, USA) projection screen $(290 \mathrm{~cm} \times 210 \mathrm{~cm})$, situated to the right of the participant.

A custom-designed activity and experience questionnaire was used to solicit information regarding levels of experience with the forehand Frisbee skill as well as with similar skills. The 20-item Reinvestment Scale (Masters et al., 1993) was also administered at the end of the experiment to ensure participants within groups were approximately matched in terms of their propensity to reinvest. We also used the 27-item Competitive State Anxiety Inventory-2 (CSAI-2; Martens et al., 1990) on two occasions; before any instructions or practice on the first day, and after providing the pressure inducing instructions, that is immediately before the stress condition assessment on the third day of testing.

After the final testing session participants were asked to recall rules/instructions provided during testing, as well as provide information as to any rules that were used that were self-generated.

\section{Procedure}

All procedures were conducted according to ethical standards of the University of British Columbia. After determining their level of skill in the forehand Frisbee throw in a pretest (five trials), participants were assigned to one of the three groups. For each practice 


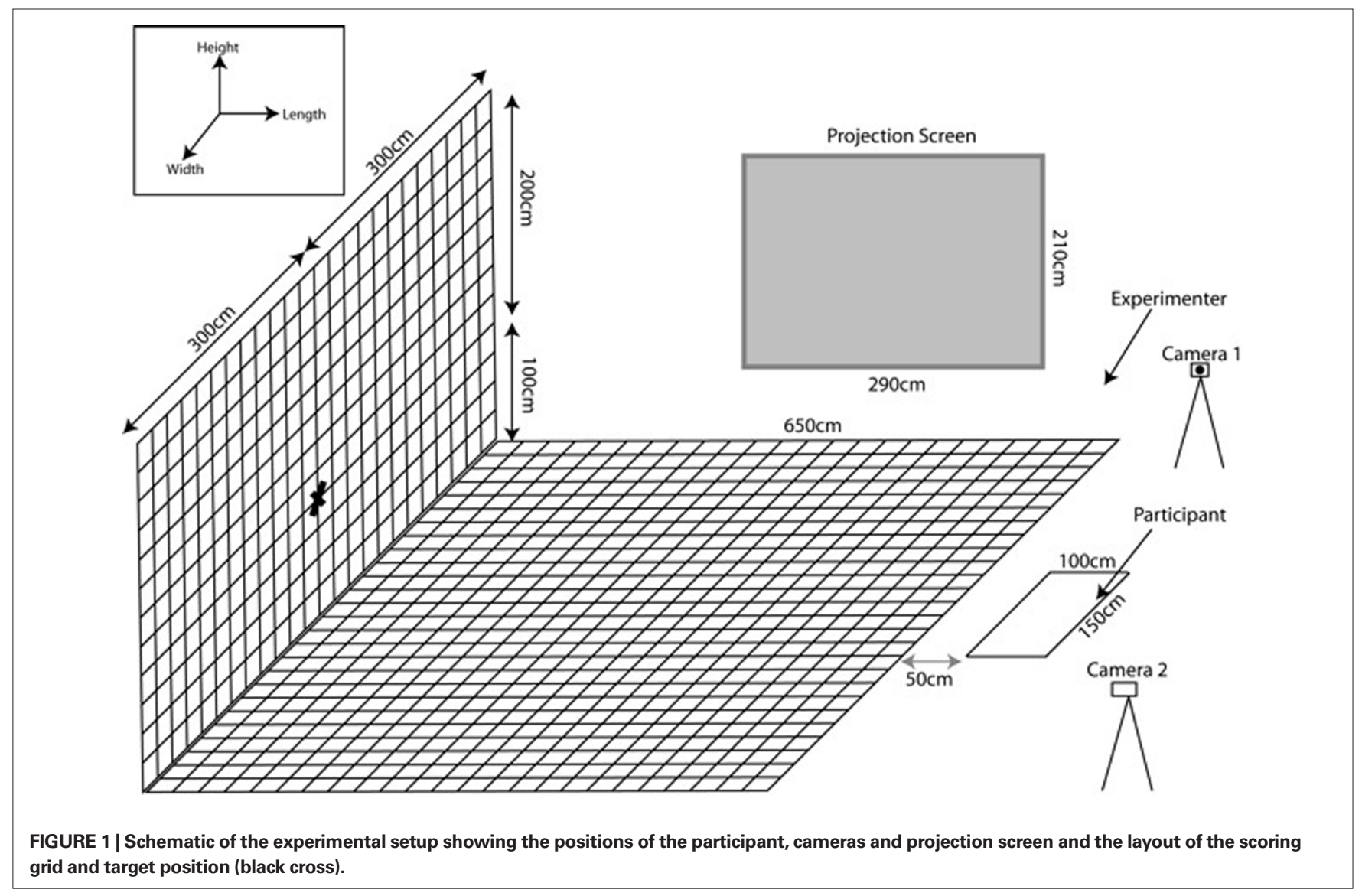

Table 1 |The eight internal-focused (Experiment 1) and external-focused (Experiment 2) instructions for the forehand Frisbee throw.

Internal-focused

\section{POSITION/STANCE}

1. Keep your body position and feet facing

forward throughout the movement or action

\section{GRIP}

2. Start with your palm up and position your hand on the disk

Hold the disk face up, grip the edge so that the

such that your thumb is on top and your index and middle fingers disk is balanced or resting on a peace sign are underneath and spaced apart

3. The web of your hand should make contact with the disk.

Position your hand out to the side $\left(90^{\circ}\right.$ angle from your body)

with the wrist slightly flexed ( $45^{\circ}$ angle)

Inner edge of the disk should make contact with

the web of your hand. Position disk out to the side $190^{\circ}$

angle) and the outer edge of the disk should hang down (45 )

DISK POSITION

4. Pull back your wrist (i.e., extend) before releasing the disk

FOOT POSITION

5. Step sideways and forward with the same foot as

the throwing arm

\section{RELEASE}

6. Snap the wrist to release the disk

7. Accelerate first your elbow and then your wrist or lead

(initiate) into the action with your elbow

8. End action with your arm straight out in front, palm facing up
Stand straight onto the target so that you are

directly facing the target during the throw
Pull back the disk before release

\section{Step into the throw}

\section{Release the disk in a snapping motion}

Release the disk as though you are whipping a horse

(like a horseman driving a horse drawn carriage) or snapping a wet towel

Imagine your hand is the disk at release so that it stays pointing up toward the target 
session on the first and second day, participants in all groups were given 40 practice trials (total of 80 trials over days 1 and 2). On the third day, there was no practice session. The INTD1 group received skill-focused instructions on the first day of practice while the INTD2 group received the same set of instructions on the second day of practice. The Control group did not receive any instruction. Participants in the INTD1 and INTD2 groups were provided with all the instructions before their respective practice sessions. These instructions were also written in large font and pinned to a wall so that they could be referred to anytime during practice. After each practice trial, participants were required to recite one of the eight instructions to the experimenter, and were reminded to follow the instructions closely and encouraged to try to improve their performance on every throw. Heart rate was measured before every trial in all experimental conditions, except during practice where it was measured at the start and before every 10th trial. A rest period of 5 min was given after 20 practice trials if participants wanted a break.

Two delayed retention conditions, each involving five trials, were administered the day following each practice session, that is, at the start of testing on day 2 and day 3. After the retention condition on day 3, participants were also assessed in a stress condition consisting of five trials. No instructions were provided during any of the retention or stress tests. To generate anxiety during the stress condition, participants were offered monetary inducements to perform well. They were informed that there would be a chance to earn an additional $\$ 10$ for the best performer in the group and that their performance would be evaluated by an expert/elite coach based on accuracy, flight path, and technique. A video camera was positioned in order to capture performance of the participant, and before the start and after each of the stress trials, participants were shown a full size image of themselves on a projection screen in order to heighten self-awareness. This image was not shown during the execution of the throw itself and hence the feedback conditions did not change. To further induce anxiety, another experimenter was introduced to the participant as an Ultimate Frisbee expert who would evaluate their performance. This person sat on a chair positioned close and to the side of the participant. She remained formal and aloof with the participant, carried a clip board with a spreadsheet in order to record performance and followed a set of pre-determined procedures before each trial in order to maintain consistency across participants. This included checking the cameras, circling the participant, moving the chair behind and to the side of the participant. These manipulations were all designed to increase the participant's belief that their technique was being evaluated by an expert.

\section{Data analysis}

Overall target error was measured as the average absolute distance between the center of the target cross on the board and the impact location of the front of the flying disk Frisbee in the $x, y$, and $z$ dimensions. This resulted in a measure of error in distance, or the $z$ dimension, that is, where the disk landed on the carpeted floor if it did not reach the board. Zero error was recorded for all throws that reached the board. Errors in width $(x)$ and height $(y)$ were recorded based on the horizontal and vertical error from the target cross respectively. If the throw landed on the floor a height error of $-100 \mathrm{~cm}$ was recorded. Error was measured to the nearest $5 \mathrm{~cm}$. From these $x, y$, and $z$ values, an overall target error was computed as the average of the absolute error in the $x, y$, and $z$ dimensions. An average of five trials was calculated to obtain an average overall target error (see Hodges et al., in press).

An instruction concordance score was calculated for each trial based on the video recording of movement form. This score was based on six observable criteria which related to the general instructions/cues that could be clearly observed during video playback. These criteria were decided in consultation among two experimenters. One point was awarded based on the appearance of each of the six criteria; body facing forward at the start of throw, elbow at $90^{\circ}$, the elbow leading the throw, a step taken during the throw, the participant stepping with the right foot, and the throw finished with the throwing arm pointing at the target. Hence, a maximum score of 6 was awarded for each trial. All trials were analyzed except for the practice trials. Analysis was conducted by one experimenter who was not naïve to the instruction conditions. However, a second rater who was naïve to the testing groups also assigned concordance scores to one randomly selected participant from each group. A positive correlation was obtained between the scores assigned by the two independent raters, $r=0.78, p<0.001$. Hence, the concordance scores assigned by the first rater were considered reliable for all further analyses.

The number of explicit instructions or cues recalled was tabulated from the debrief interview. Participants could potentially list a maximum of 12 cues (see Table 1, left column in bold) that were derived from the set of eight instructions. The number of cues recalled was correlated with reinvestment scores, as well as average overall target error during the stress condition to assess if the amount of explicit knowledge about throwing was moderated by the individual's disposition to reinvest as well as whether it was related to performance under pressure. Pearson product moment correlations were calculated.

Mixed-factor analyses of variance (ANOVA) were conducted for the dependent variables; overall target error, concordance, number of cues recalled, heart rate, and CSAI. For the CSAI only the cognitive anxiety-state and somatic anxiety-state subscales were analyzed (see Liao and Masters, 2002; Wang et al., 2004). Group (INTD1, INTD2, Ctrl) was the between-subjects' factor, while Day (day 1, day 2, day 3), Condition (practice, delayed retention, stress), Time (early or late in practice), and/or Block (each block consisting of five trials) were within-subject factors. Partial eta squared $\left(\eta_{p}^{2}\right)$ values were reported as measures of effect size and post hoc analyses were conducted using Tukey HSD procedures $(p<0.05)$.

\section{RESULTS}

\section{Overall target error}

Average overall target error as a function of group, day, condition, and practice block is illustrated in Figure 2. A one-way ANOVA comparing across the three groups (INTD1, INTD2, and Ctrl) during the pretest showed that they were not significantly different, $F<1$.

We compared the first two blocks and last two blocks of practice over the 2 days of practice. As a result of improvements in accuracy across time and with the accumulation of practice, main effects were found for day, $F(1,26)=8.41, p<0.01, \eta_{p}^{2}=0.24$, time, 


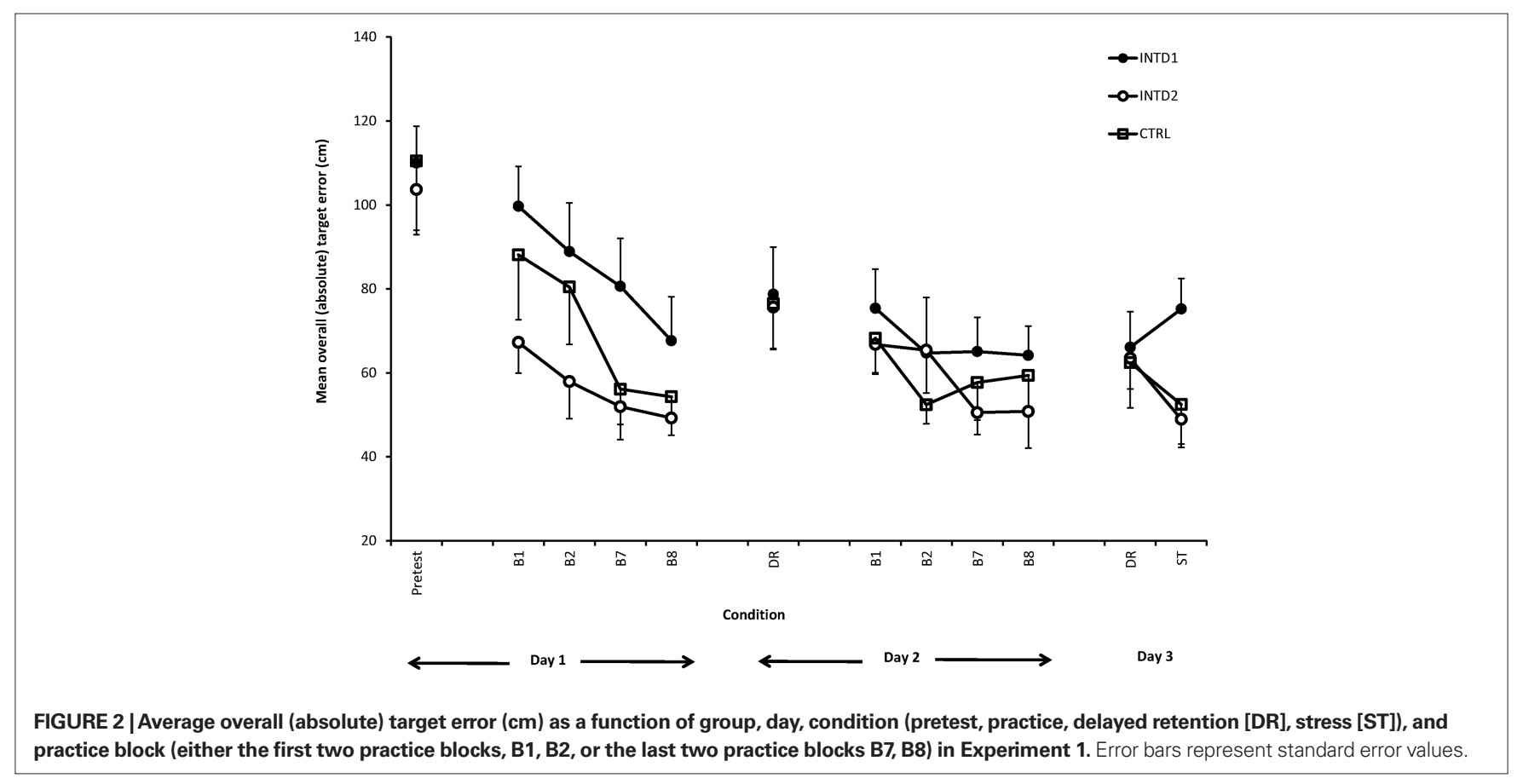

$F(1,26)=22.68, p<0.001, \eta_{p}^{2}=0.47$, and block, $F(1,26)=6.69$, $p<0.05, \eta_{p}^{2}=0.21$. Although there was no main effect of group, a significant Group $\times$ Day interaction was observed, $F(2,26)=3.64$, $p<0.05, \eta_{\mathrm{p}}^{2}=0.22$. As can be seen in Figure 2 and confirmed by post hoc analysis, on day 1 of practice the INTD 1 instruction group $(M=84.21, \mathrm{SD}=34.79 \mathrm{~cm})$ showed more error in comparison to the INTD2, no-instruction group $(M=56.58, \mathrm{SD}=23.12 \mathrm{~cm})$. The control group was not significantly different from either group. The INTD1 group was also the only group to significantly reduce their error from day 1 to day 2 of practice $(M=67.33$, $\mathrm{SD}=29.89 \mathrm{~cm}$ ).

A 3 Group $\times 2$ Day ANOVA was conducted on the delayed retention conditions on day 2 and day 3. There was a main effect of day, $F(1,26)=9.01, p<0.01, \eta_{p}^{2}=0.26$. The groups were more accurate in their performance during day $3(M=64.03, \mathrm{SD}=26.51 \mathrm{~cm})$ than day $2(M=76.97, \mathrm{SD}=31.96 \mathrm{~cm})$. There were, however, no effects involving group, $F s<1$.

To compare the effect of a pressure inducing condition on the groups, a one-way ANOVA was conducted for the stress condition on day 3. The group effect was significant, $F(2,26)=3.35, p=0.05$, $\eta_{p}^{2}=0.21$.. This was a result of the higher error for the INTD1 group in comparison to the INTD2 group.

\section{Instruction concordance}

Because the Control group did not receive any instructions, they were not assigned concordance scores. In order to assess change in movement form as a function of the instruction conditions and to enable inferences as to whether participants were using the instructions, we calculated a performance change score. This was obtained for practice on day 1 by subtracting the average concordance score obtained during the five pretest trials from the average score obtained during the five trials of delayed retention 1 (on day 2). For practice on day 2 , this score was calculated by subtracting the average concordance score of delayed retention 1 (day 2) from delayed retention 2 (day 3 ). These data are illustrated in Figure 3.

A 2 Group (INTD1, INTD2) × 2 Day (day 1, day 2) ANOVA was conducted on these change scores. As would be predicted if the groups were using the instructions the Group $\times$ Day interaction was significant, $F(1,18)=18.20, p<0.001, \eta_{\mathrm{p}}^{2}=0.50$. Post hoc analysis confirmed that the mean difference in instruction concordance, as inferred through changes in movement form across practice days, was significantly greater for the INTD1 group $(M=+1.88$, $\mathrm{SD}=1.35)$ than the INTD2 group $(M=+0.58, \mathrm{SD}=1.26)$ after practice on day 1 . As expected, after practice on day 2 this observation was reversed (INTD1: $M=-0.50, \mathrm{SD}=1.41$; INTD2: $+M=1.48$, $\mathrm{SD}=0.90)$.

\section{Recall of instructions}

There was a significant difference between the groups in the number of explicit cues recalled (for INTD1 and INTD2 groups) and /or self-generated (for Ctrl group), $F(2,26)=14.54, p<0.001, \eta_{\mathrm{p}}^{2}=0.53$. The INTD1 group $(M=6.3, \mathrm{SD}=2.2)$ and INTD2 group $(M=7.0$, $\mathrm{SD}=1.9)$ did not differ in terms of the number of cues recalled, but they both recalled significantly more instructions than the Ctrl group $(M=2.4, \mathrm{SD}=1.7)$.

\section{Stress check manipulations}

CSAI-2. We analyzed the summated scores from the cognitive anxiety-state and the somatic anxiety-state subscales obtained from the CSAI-2 administered before instructions and practice on day 1 , and between stress instructions and execution on day 3. The means are presented on the left side of Table 2. There was a general increase in perceived anxiety just before the stress test $(M=30.0)$, in comparison to anxiety levels before the commencement of testing on day $1(M=28.6)$, but the condition 


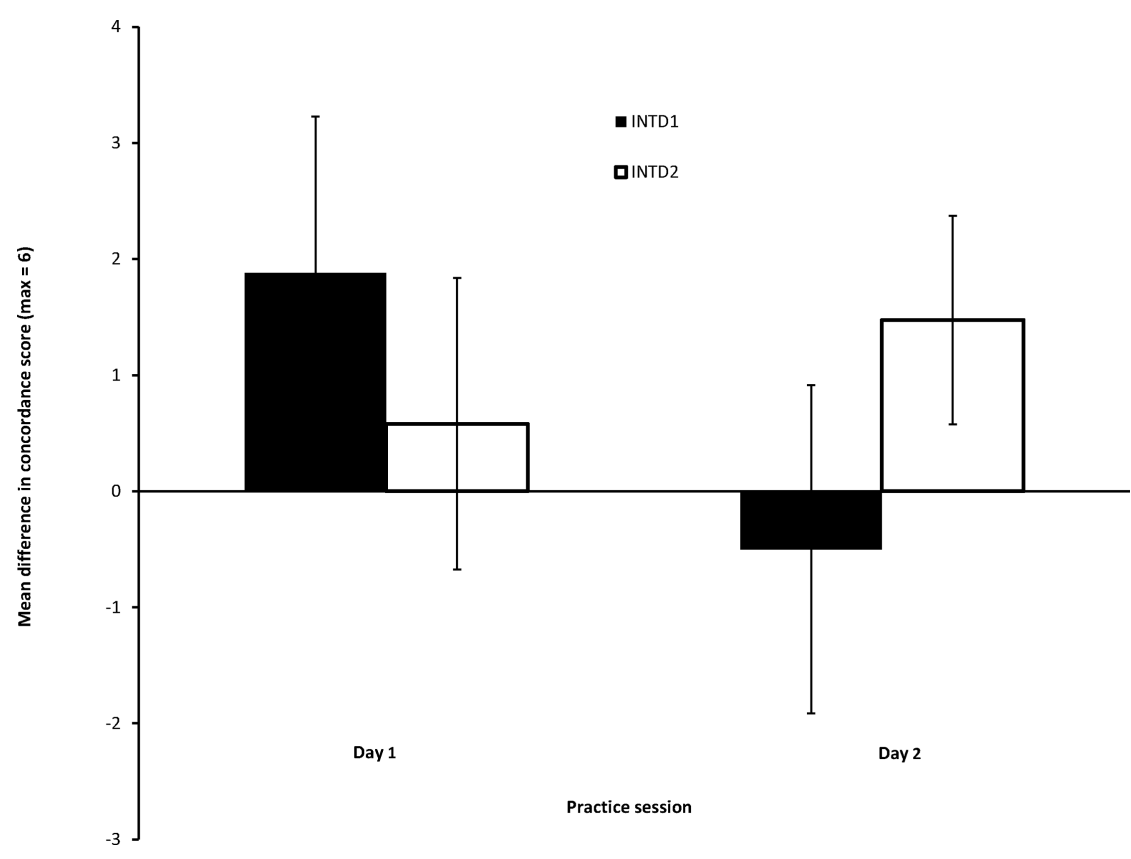

FIGURE 3 | Mean difference in instruction concordance scores for the two internal instruction groups (INTD1 and INTD2) after day 1 and day 2 of practice.

Table 2 | Average heart rate data (bpm) and scores on the cognitive and somatic subscales of the CSAI-2 (and SDs) as a function of group and testing day and/or condition across the two experiments.

\begin{tabular}{llll}
\hline Experiment/group & $\begin{array}{l}\text { Day 1_Start } \\
\text { CSAl }\end{array}$ & Day 3_Stress & $\begin{array}{l}\text { End_Day 2 } \\
\text { HR }\end{array}$
\end{tabular}

\begin{tabular}{|c|c|c|c|c|c|}
\hline \multicolumn{6}{|c|}{ EXPERIMENT 1} \\
\hline Internal Day 1 & $31.7(5.6)$ & $30.8(9.0)$ & 94.9 (11.6) & $95.5(13.7)$ & 98.8 (11.6) \\
\hline Internal Day 2 & $27.2(6.7)$ & 30.8 (8.5) & 93.1 (10.6) & 95.3 (9.9) & $95.3(13.2)$ \\
\hline Overall means & $28.6(5.8)$ & $30.0(7.4)$ & $92.6(10.2)$ & $95.4(11.2)$ & $97.0(11.4)$ \\
\hline \multicolumn{6}{|c|}{ EXPERIMENT 2} \\
\hline External Day 1 & $26.1(3.7)$ & $27.8(5.8)$ & $85.3(16.0)$ & $92.2(16.7)$ & $91.0(17.4)$ \\
\hline
\end{tabular}

effect was not significant, $F(1,26)=1.80, p=0.19, \eta_{\mathrm{p}}^{2}=0.07$. When specifically asked to comment on whether they perceived their heart to be racing due to increased anxiety, just before they began to throw in their respective conditions, there was a general increase in this perception from the start of testing on day 1 to day 3 (Ms $=1.34-1.51)$, but the day effect was not significant, $F(1,26)=2.77, p=0.11, \eta_{\mathrm{p}}^{2}=0.10$.

Heart rate. As can be seen from the top half of Table 2 (right side), there was a general increase in heart rate across the two testing conditions on day 3 (before retention, $M=95.4 \mathrm{bpm}$; before stress test, $M=97.0 \mathrm{bpm}$ ). When analyzed in a 3 Group $\times 2$ Condition ANOVA this difference was not statistically significant, $F(1,26)=3.06, p=0.09, \eta_{\mathrm{p}}^{2}=0.11$. No group effects were observed. In view of the fact that the retention test and measures of $\mathrm{HR}$ were collected as soon as participants entered the laboratory, and were likely elevated as a result of travel to testing, we compared the HR values in the stress test to the last five trials of HR data recorded on the second day of practice $(92.6 \mathrm{bpm})$. There was a significant increase in HR during the stress test, $F(1,26)=5.56$, $p<0.05, \eta_{\mathrm{p}}^{2}=0.18$.

Debrief. Six participants in the INTD2 group and five participants in each of the other groups spontaneously commented that they found the competitive/evaluative conditions on day 3 stressful or that they were nervous on this final test on day 3, when asked to comment generally on their experiences over the 3 days of practice (this number did not include responses from participants who claimed that the test buoyed them up, or helped make them feel more motivated or competitive). 


\section{Reinvestment data}

The groups did not differ in terms of the propensity to reinvest, $F<1$ (INTD1: $M=3.81, \mathrm{SD}=0.77$; INTD2: $M=4.02, \mathrm{SD}=1.10$; Ctrl: $M=3.77, \mathrm{SD}=1.11)$. There was no indication that the reinvestment scores correlated with the number of explicit instructions (cues) recalled, $r=0.03, p>0.05$.

\section{DISCUSSION}

We examined how the timing of internally focused instructions to novice Frisbee players moderates performance in retention and under stress-inducing conditions. Two groups were compared who received the same amount of technical, internally focused instruction, but it was received either on day 1 or day 2. These groups were also compared to a third group who did not receive any technical instruction. We predicted different performance outcomes during the stress-inducing condition as a function of group, based on differential predictions of the reinvestment hypothesis and the skill-focused attention theory. If (internally focused)instructions serve to promote a more conscious, controlled mode of performing, then according to the skill-focused attention theory we would not expect to see performance breakdowns as a result of stress for the INTD2 group. This is due to the fact that the group who receives internally focused instructions on the second day would be performing under similar conditions as expected to be prompted by stress-inducing situations (i.e., conscious and controlled). In contrast, if providing instructions later in practice serves to make these instructions more salient and more accessible to consciousness, then according to the reinvestment hypothesis we would expect to see breakdowns in the INTD2 group under pressure.

What we found was that the INTD2 group was not negatively affected by the stress manipulation, only the INTD1 group was. This finding lends support to the skill-focused attention theory, where it is believed that the cognitive processes encouraged during practice (either more controlled or automatic in nature), dictate performance under pressure. Accordingly, only participants who were performing in a more "automatic" or proceduralized manner (which we would expect for the INTD1 group), were expected to show performance breakdowns under pressure. Because there was no evidence that the groups differed in propensity to reinvest and in terms of the amount of instruction they recalled, there was no evidence that the instructions were more salient for the INTD2 group and hence no reason for these individuals to show a greater propensity to reinvest during the stress-inducing condition. Importantly, however, because of the differential performance of the two instruction groups it does seem that it is not merely the amount of instruction that is important in predicting performance under anxiety-inducing conditions but also when in practice this is provided.

There was evidence that both groups used the instructions they were given to improve their technique as demonstrated by the change in movement form or mean difference in concordance score as a function of practice. Providing internally focused instructions to the INTD1 group on the first day of practice significantly affected how they performed the Frisbee throw. However, they were not able to use the instructions to improve outcome performance on the first day of practice, as evidenced by their greater target error in comparison to the INTD2 group. This differential performance as a function of instruction, with instructions serving to have an adverse effect on outcome success, has been shown elsewhere. For example, Vereijken (1991) showed that performance on a ski-simulator was negatively affected as a result of technical instruction. Similar results have also been seen when participants have been required to learn novel bimanual coordination movements and in all these examples the instructions were focused on body-related technical features (see Hodges and Lee, 1999; Hodges and Franks, 2000).

Because in this experiment the instructions were always internally directed, it is possible that the negative effects associated with providing how-to instructions on the first day (i.e., during practice and under stress-inducing conditions) were a result of the direction of attention encouraged by these instructions, a prediction we explore in Experiment 2 (see Wulf and Prinz, 2001; Wulf, 2007a,b). It is important to point out, however, that because the INTD1 group was not more errorful than the control group during the delayed retention tests, these instructions were not detrimental for learning under normal conditions and the instructions did have a positive impact on change in movement form.

\section{EXPERIMENT 2 INTRODUCTION}

Ina second Experiment, we examined whether a change in the focus of the instructions, from an internal, body-related focus, to an external, effects-related focus, moderates the effects associated with when in practice instructions are provided and particularly performance under stress-inducing conditions. Because externally directed instructions were not expected to prompt an explicit, conscious mode of processing, we did not expect differences between these two groups in terms of performance under stress. If the accrual of explicit instruction in general leads to performance decrements under stress-inducing conditions, and there was evidence that these external instructions were recalled by both groups (and more so by the EXTD2 group), we would expect performance detriments in both groups under stress (reinvestment hypothesis). However, if participants have not accrued explicit information about how to perform and have instead learned in a more "automatic" type of way, then under stress they would not revert to a conscious mode of performing as this never defined their performance (skillfocused attention).

\section{METHOD \\ Participants}

Twenty, right-hand dominant participants (age range: 18-25 years) were newly recruited and quasi-randomly assigned to either the External Day 1 (EXTD1; $n=10 ; 9$ females; mean age $=22$ years), or the External Day 2 (EXTD2, $n=10 ; 10$ females; mean age $=21$ years) group so that the two groups were approximately matched on pretest average overall absolute error. As with Experiment 1, participants had no previous or only recreational Frisbee playing experience and met the pretest criteria for inclusion (two participants were excluded before group allocation). Informed consent was obtained from all participants and participant recruitment and procedures were approved and met the standards of the University of British Columbia's Behavioral Research Ethics' Board. 


\section{Task goals, materials, and apparatus}

These were identical to those detailed in Experiment 1, except participants received a set of eight external-focused instructions (12 external cues) that described the same technique as the internally focused instructions or cues (see right side of Table 1).

\section{Procedure}

The procedures were identical to those adopted in Experiment 1 . The EXTD1 group read and recited external-focused instructions only during practice on day 1 , while the EXTD2 group received the same instructions only during practice on day 2 .

\section{Data analysis}

The analyses were mostly comparable to those adopted in Experiment 1, with the exception that now only two groups were compared.

\section{RESULTS}

\section{Overall target error}

The average overall target error for the external-focused groups in all conditions is illustrated in Figure 4. The two external groups were not significantly different on pretest performance, $t(18)=0.78$, $p>0.05, d=0.37$.

Both groups improved across practice as evidenced by main effects for day, $F(1,18)=19.33, p<0.001, \eta_{\mathrm{p}}^{2}=0.52$, time, $F(1,18)=18.85, p<0.001, \eta_{\mathrm{p}}^{2}=0.51$, and a Day $\times$ Block interaction, $F(1,18)=6.75, p<0.05, \eta_{\mathrm{p}}^{2}=0.27$. On the first day, regard- less of whether it was the beginning or end of each practice session, the second block of practice was more accurate than the first block. There was no significant difference in accuracy between the two practice blocks on day 2. A main effect for Group was also observed, $F(1,18)=4.76, p<0.05, \eta_{p}^{2}=0.21$. The EXTD1 group $\left(M_{\text {error }}=62.54, \mathrm{SD}=36.32 \mathrm{~cm}\right)$ was more accurate than the EXTD2 group $\left(M_{\text {error }}=85.20, \mathrm{SD}=36.10 \mathrm{~cm}\right)$, irrespective of practice day, and there were no further interactions involving group.

When performance during delayed retention on day 2 and day 3 was analyzed, only a main effect of Day, $F(1,18)=10.95, p<0.01$, $\eta_{\mathrm{p}}^{2}=0.38$, was observed. Outcome errors decreased across retention sessions (day 2: $M=89.38, \mathrm{SD}=42.12 \mathrm{~cm}$; day $3: M=62.37$, $\mathrm{SD}=20.02 \mathrm{~cm})$. Despite the differences in error in the first retention test, the group effect was not significant, $F(1,18)=2.17, p=0.16$, $\eta_{\mathrm{p}}^{2}=0.11$, neither was the Group $\times$ Day, $F=1$. A $t$-test comparing group means during the stress condition on day 3 did not yield a significant group difference, $t(18)=0.96, p>0.05, d=0.45$.

\section{Concordance}

Again we looked at the mean difference in concordance scores as a function of practice sessions as shown in Figure 5. Only the twoway interaction of Group $\times$ Day was significant, $F(1,18)=17.93$, $p<0.001, \eta_{\mathrm{p}}^{2}=0.50$. As shown in the Figure and confirmed by post hoc analysis, after practice on day 1, only the EXTD1 group showed a significant change in movement form in accordance with the instructions (EXTD1: $M=+1.82, \mathrm{SD}=1.56$; EXTD2: $M=-0.54$,

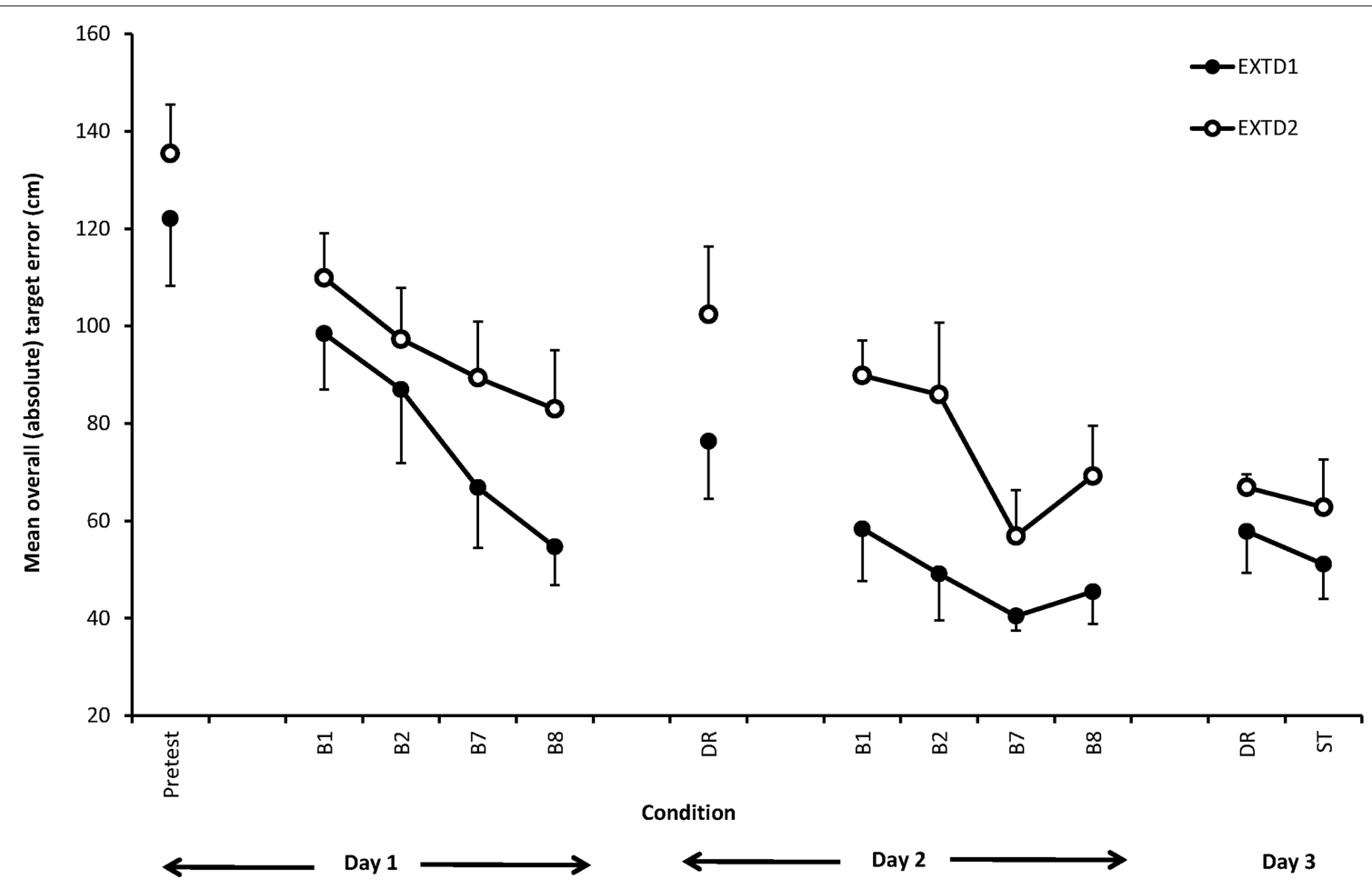

FIGURE 4 | Average overall (absolute) target error (cm) as a function of group, day, condition (pretest, practice, delayed retention [DR], stress [ST]), and practice block (either the first two practice blocks, B1, B2, or the last two practice blocks B7, B8) in Experiment 2. Error bars represent standard error values. 
$\mathrm{SD}=1.33)$. This observation was reversed for practice on day 2 (EXTD1: $M=+0.04, \mathrm{SD}=0.26$; EXTD2: $M=+1.94, \mathrm{SD}=1.65$ ). The improvement in concordance score after practice with instructions indicated that participants used the instructions provided to improve their throwing technique.

\section{Recall of instructions}

The average number of cues recalled by the EXTD1 group was 8.0 $(\mathrm{SD}=2.3)$, and for the EXTD2 group it was $7.5(\mathrm{SD}=2.2)$, these were not significantly different, $t(18)=0.63, p>0.05$. The number of cues recalled did not correlate with target error during the stress condition, $r=-0.22, p>0.05$.

\section{Stress check manipulations}

CSAI-2. These data are illustrated in the bottom left of Table 2. Although there was an increase in scores across the 2 days (before instructions and practice on day 1 , and between instructions and execution on day 3 ), the condition effect was not significant, $F(1,18)=2.04, p=0.17, \eta_{\mathrm{p}}^{2}=0.10$. None of the effects involving group were significant. When participants were asked to comment on whether they perceived their heart to be racing due to increased anxiety, there was a significant increase in their perception from the start of testing on day 1 to before the stress test on day 3 $(M s=1.15-1.55), F(1,26)=8.47, p<0.01, \eta_{p}^{2}=0.32$.

Heart rate. These data can be viewed in the bottom of Table 2 (right side). Based on a 2 Group $\times 2$ Condition (day 3 retention and stress test) ANOVA, no significant differences were found as a function of group or condition (all Fs $<1$ ). As with Experiment 1, we compared the HR collected in the last five recorded trials of day 2 and compared these to the stress test HR values. HR increased from 86.4 to $92.7 \mathrm{bpm}, F(1,18)=4.31, p=0.052, \eta_{\mathrm{p}}^{2}=0.19$.

Debrief. Five participants in each group spontaneously commented that they were nervous or stressed on the third day of testing when they were being evaluated, when asked to comment generally on their experiences over the 3 days of practice.

\section{Reinvestment data}

The groups did not differ in terms of the propensity to reinvest, $t(18)=1.08, p>0.05, d=0.51($ EXTD $1: M=3.63, \mathrm{SD}=1.20$; EXTD2: $M=4.27, \mathrm{SD}=1.44)$. There was no indication that the reinvestment scores correlated with the number of explicit instructions recalled, $r=0.19, p>0.05$.

\section{DISCUSSION}

In this experiment, we did not expect the instructions to promote a conscious controlled mode of processing and hence we would not expect a reversion to a previous conscious mode of control in either group under pressure-induced conditions. Our data supports this conclusion. Both groups showed resistance to the phenomenon of choking under pressure. Even though both groups were able to recall a significant number of the instructions given, this explicit knowledge did not detrimentally affect performance under stressful conditions. Therefore, contrary to strict ideas of the reinvestment hypothesis, learning in an explicit rule-based manner does not lead to reinvestment of this knowledge under pressure in a negative way when the rules acquired are externally

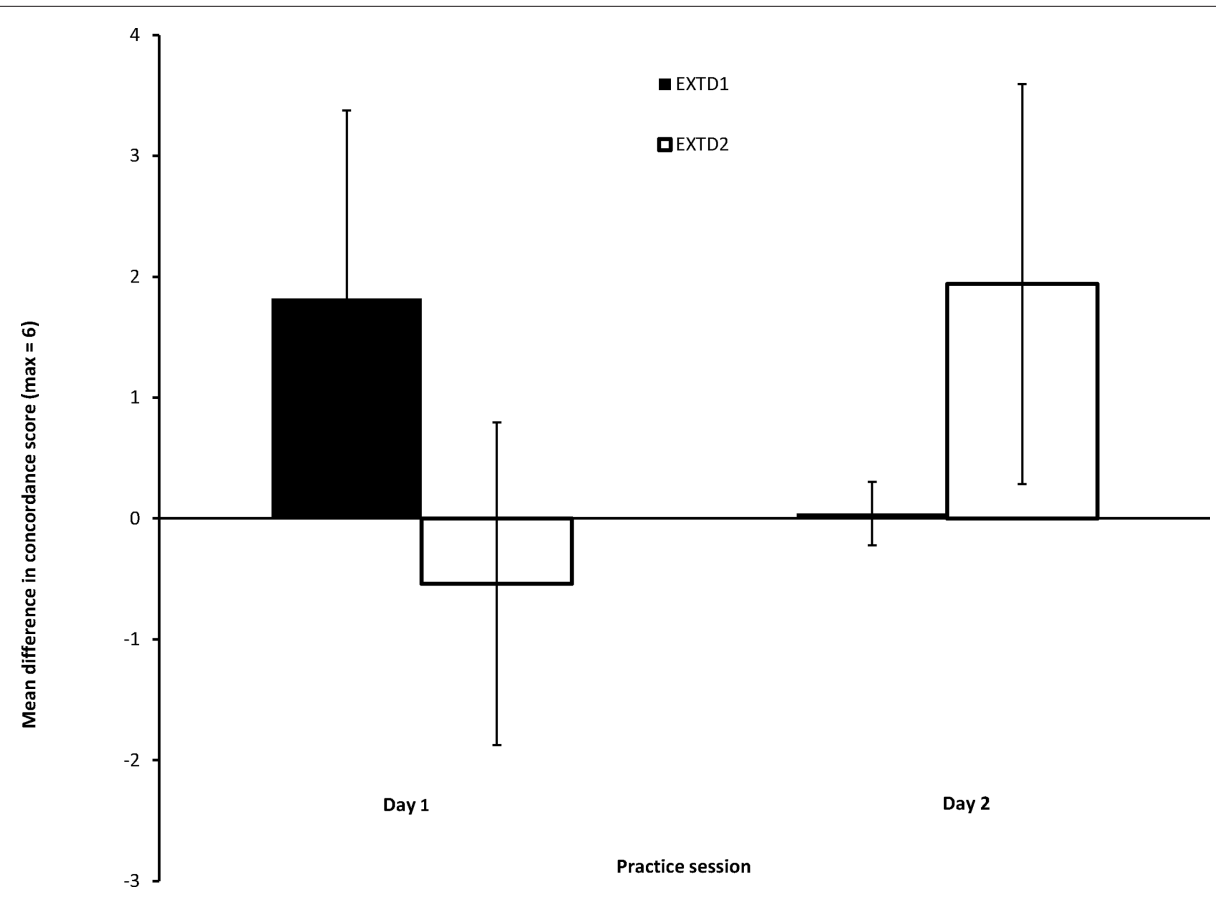

FIGURE 5 | Mean difference in instruction concordance scores for the two external instruction groups (EXTD1 and EXTD2) after day 1 and day 2 of practice. 
directed. It would appear that the externally directed instructions encouraged a more implicit/automatic mode of learning right from the beginning. Even though the EXTD2 group did not receive instructions until day 2, there was no evidence (similar to Experiment 1) that these control, no instruction conditions, prompted a more conscious, explicit mode of learning which negatively impacted performance under stress when performance was assessed on day 3 .

As in Experiment 1, both externally focused groups used the instructions in order to improve performance as evidenced in the change in concordance scores as a function of practice session and group. The EXTD1 group showed early advantages associated with the provision of these instructions in comparison to the non-instructed group on the first day (i.e., EXTD2). This result is qualitatively different to what we saw for the internalfocused instructions groups, where the no-instruction conditions resulted in lower error (at least during practice) in comparison to the internally focused instructions (INTD1). Although we did not make direct quantitative comparisons across the internal and external groups (because of minor differences in testing procedures, differences in when they were tested and higher pretest values for the two external groups), these results support previous literature showing that in comparison to externally directed instructions, internally focused instructions can detrimentally affect skill acquisition (see Wulf and Prinz, 2001; Wulf, 2007a,b, for reviews).

However, one potentially important caveat with respect to the statement above concerns the method of instruction used to convey the external-focused instructions. Two of our eight external-focused instructions ("resting on a peace sign" and "whipping a horse") were based on analogies, a method of instruction that is thought to encourage a more implicit type of motor learning, by reducing the amount of information that is consciously processed by the performer (Masters and Maxwell, 2004). Although it is possible that the amount of information conveyed by our external-focused instructions was reduced compared to our internal-focused instructions (at least for 2 of the instructions), the externally directed groups recalled approximately eight cues, which was comparable to in the seven for the internal groups. Such analogy instructions have similarly been used to encourage an external focus of attention in previous research (e.g., Wulf et al., 1999), suggesting that the benefits of these types of analogy instructions goes beyond the amount of information.

In summary, when instructions are focused on external movement effects, no performance breakdowns as a function of pressure are seen and the timing of instruction provision does not affect how learners perform in later testing (either with or without external pressures). Therefore, it would seem that the reinvestment hypothesis only applies when instructions are provided that are internal, or body-focused in nature. In other research (Ford et al., 2005; Castaneda and Gray, 2007) it has been shown that instruction effects are moderated by the direction of attention encouraged by instructions. The results from the current experiments underscore these findings showing that performance effects are influenced by the attentional focus encouraged by the instructions and not merely whether the instructions are skill-focused (cf., Beilock et al., 2002).

\section{GENERAL DISCUSSION}

When providing technical, internally focused instructions, learners benefit more under pressure if instructions are provided "later" in practice. Promoting self monitoring and conscious control during practice right before performing under pressure was shown to help prevent performance decrements in Experiment 1, which concurs with the skill-focused attention theory. However, because of the benefits seen in the control participants who did not receive instruction on either day, it is more likely that withholding instructions early in practice promotes a less conscious/ explicit mode of practice than that encouraged by (internal) instructions and that irrespective of whether instructions are provided later, this initial uninstructed practice serves to inoculate the performer somewhat against later pressure situations. This hypothesis is supported by findings from Experiment 2. Manipulations to the timing of externally focused instructions did not impact performance under pressure. Both external-focused groups showed no adverse reactions to the stress condition. It appears that this manner of learning, one that encourages a more "automatic" mode of control (McNevin and Wulf, 2002) despite the fact that rule-based instructions were provided, helps to prevent disruptions under pressure. Rather than the amount of instruction and the skill focus encouraged by these instructions causing disruptions and reinvestment under pressure (as predicted by the reinvestment hypothesis and skill-focused attention theory), it appears that the effects of instructions are moderated by the attentional focus encouraged by these instructions (see Ford et al., 2005; Castaneda and Gray, 2007). These results add further support to the prevailing evidence that an external focus of attention is more effective than an internal focus, in terms of protecting against the potentially harmful effects associated with pressure and competition.

The current experiments are, however, faced with a few limitations. First, we did not find statistically significant indications of increased stress in all measures across both experiments. This was surprising given that in previous studies (such as Masters, 1992), similar evaluative conditions and monetary incentives led to significant increases in anxiety. In addition to these previous scenarios, we also adopted a video monitoring condition (see Beilock and Carr, 2001; Gray, 2004) and the evaluation was conducted by a live person. However, across practice days and retention/stress tests on day 3 , there was evidence that HR had increased and half of the participants in both experiments spontaneously remarked during the debrief on how stressful the last testing session was (especially the evaluative presence of the second person). Further, the stress condition did serve to differentiate the groups in terms of outcome error in Experiment 1 only, in accordance with predictions. It is perhaps not surprising that participants had an elevated HR when first arriving in the laboratory for testing on the final day, both because of the uncertainty of the testing conditions and because of physical exertion required to get to the laboratory. In future experiments it would be important to control for these confounding factors when measuring heart rate before and after a manipulation and to directly question participants on their experiences in these conditions after the experiment to further gage their validity. There are always challenges associated with simulation and measurement of stress or pressure under laboratory conditions, not least because real costs or rewards associated with performing 
within the field can never truly be simulated under contrived conditions (see Gucciardi and Dimmock, 2008) and participants have little investment in the results of their performance.

As discussed earlier, in both experiments, the timing of instructions did not moderate the number of rules that could be recalled about how to perform the throw. Therefore, it could be argued that the rules were not more salient on the days when they were given, despite the fact that these rules/instructions were given verbally, posted on a wall which could be studied in between trials, and participants were asked to recite one of the rules before each practice attempt on the days when they were provided. This constant rehearsal of instructions (cf. Masters, 1992 whereby written instructions were only provided in a 5 -min period before practice on each day) might have made it easier to recall instructions regardless of when in practice they were provided.

In conclusion, although the current results do not refute the reinvestment hypothesis related to choking, there is evidence that the timing of instructions is an important factor in skill learning, such that withholding internally focused, technical instruction, until a certain level of proficiency and familiarization has been

\section{REFERENCES}

Anderson, J. R. (1982). Acquisition of cognitive skill. Psychol. Rev. 89, 369-406.

Anderson, J. R. (1983). The Architecture of Cognition. Cambridge, MA: Harvard University Press.

Baumeister, R. F. (1984). Choking under pressure: self-consciousness and paradoxical effects of incentives on skilful performance. J. Pers. Soc. Psychol. 46, 610-620.

Baumeister, R. F., and Steinhilber, A. (1984). Paradoxical effects of supportive audiences on performance under pressure: the home field disadvantage in sports championships. J. Pers. Soc. Psychol. 47, 85-93.

Beilock, S. L., and Carr, T. H. (2001). On the fragility of skilled performance: what governs choking under pressure? J. Exp. Psychol. Gen. 130, 701-725.

Beilock S. L., and Carr, T. H. (2004). "From novice to expert performance: memory, attention and the control of complex sensori-motor skills," in Skill Acquisition in Sport, Research, Theory and Practice, eds A. M. Williams and N. J. Hodges (London: Routledge), 207-228.

Beilock, S. L., Carr, T. H., MacMahon, C., and Starkes, J. L. (2002). When paying attention becomes counterproductive: impact of divided versus skill-focused attention on novice and experienced performance of sensorimotor skills. J. Exp. Psychol. Appl. 8, 6-16.

Bennet, S. J. (2000). Implicit learning: should it be used in practice? Int. J. Sport Psychol. 31, 542-546.

Castaneda, B., and Gray, R. (2007). Effects of focus of attention on baseball bat-

attained, acts to prevent possible detrimental effects associated with pressure situation. This lends support to the skill-focused attention theory of choking. However, we showed in a second experiment that if these instructions were externally directed, then no negative consequences associated with instructions early or later in practice under stress-inducing conditions were seen. In view of the fact that technical instructions are viewed to be important by coaches to aid with self correction of errors (see Bennet, 2000; Hill et al., 2010) and that we have evidence that movement form improves following these types of instruction, then these results are especially important in providing advice as to when to provide instructions and how to direct attention appropriately to warn off any potentially negative learning/performance effects.

\section{ACKNOWLEDGMENT}

The final author (Nicola J. Hodges) would like to acknowledge the support of the Social Sciences and Humanities Research Council of Canada (SSHRC) who provided operating grants that funded this research.

to learning a bimanual visual-motor coordination task: do instructions of the movement pattern facilitate learning relative to discovery learning. Br. J. Psychol. 90, 389-403.

Kimble, G.A., and Perlmuter, L. C. (1970). The problem of volition. Psychol Rev., 77, 361-384.

Liao, C. M., and Masters, R. S. W. (2002). Self-focused attention and performance failure under psychological stress. J. Sport Exerc. Psychol. 24 289-305.

Lewis, B. P., and Linder, D. E. (1997) Thinking about choking? Attentional processes and paradoxical performance. Pers. Soc. Psychol. Bull., 23 937-944.

Martens, R., Vealey, R., and Burton, D. (1990). Competitive Anxiety in Sport. Champaign, IL: Human Kinetics.

Masters, R. S. W. (1992). Knowledge, knerves and know-how: the role of explicit versus implicit knowledge in the breakdown of a complex motor skill under pressure. Br. J. Psychol. 83 , 343-358.

Masters, R., and Maxwell, J. (2008). The theory of reinvestment. Int. Rev. Sport Exerc. Psychol. 1, 160-183.

Masters, R. S. W., and Maxwell, J.P. (2004) "Implicit motor learning, reinvestment and movement disruption: what you don't know won't hurt you," in Skill Acquisition in Sport, Research, Theory and Practice, eds A. M. Williams and N. J. Hodges (London: Routledge), 207-228.

Masters, R. S. W., Polman, R. C. J., and Hammond, N. V. (1993). 'Reinvestment': a dimension of personality implicated in skill breakdown under pressure. Pers. Individ. Dif. 14 655-666.

Maxwell, J. P., Masters, R. S. W., and Poolton, J. M. (2006). Performance breakdown in sport: the roles of reinvestment and verbal knowledge. Res. Q. Exerc. Sport 77, 271-276.

McNevin, N. H., and Wulf, G. (2002). Attentional focus on supra-postural tasks affects postural control. Hum. Mov. Sci. 21, 187-202.

Schneider, W., and Shiffrin, R. M. (1977) Controlled and automatic human information processing: I. Detection, search and attention. Psychol. Rev. 84, $1-66$.

Totsika, V., and Wulf, G. (2003). The influence of external and internal foci of attention on transfer to novel situations and skills. Res. Q. Exerc. Sport 74, 220-226.

Vereijken, B. (1991). The Dynamics of Skill Acquisition. Meppel: Krips Repro.

Wang, J., Marchant, D., Morris, T., and Gibbs, P. (2004). Self-consciousness and trait anxiety as predictors of choking in sport. J. Sci. Med. Sport, 7, 174-185

Wulf, G. (2007a). Attentional focus and motor learning: a review of 10 years of research. Bewegung Train. 1, 4-14.

Wulf, G. (2007b). Attention and Motor Skill Learning. Champaign, IL: Human Kinetics.

Wulf, G., Hoss, M., and Prinz, W. (1998). Instructions for motor learning: differential effects of internal versus external-focus of attention. J. Mot. Behav. 30, 169-179.

Wulf, G., Lauterbach, B., and Toole, T. (1999). The learning advantages 
of an external focus of attention in golf. Res. Q. Exerc. Sport 70, 120-126.

Wulf, G., McConnel, N., Gärtner, M., and Schwarz, A. (2002). Feedback and attentional focus: enhancing the learning of sport skills through external-focus feedback. J. Mot. Behav. 34, 171-182.

Wulf,G.,McNevin, N., and Shea,C.H.(2001). The automaticity of complex motor skill learning as a function of attentional focus. Q. J. Exp. Psychol. 54, 1143-1154.

Wulf, G., and Prinz, W. (2001). Directing attention to movement effects enhances learning: a review. Psychon. Bull. Rev., 8, 648-660.

Conflict of Interest Statement: The authors declare that the research was conducted in the absence of any commercial or financial relationships that could be construed as a potential conflict of interest.

Received: 01 September 2010; accepted: 21 October 2010; published online: 16 November 2010.

Citation: OngNT, Bowcock A and Hodges NJ (2010) Manipulations to the timing and type of instructions to examine motor skill performance under pressure. Front. Psychology 1:196. doi: 10.3389/fpsyg.2010.00196
This article was submitted to Frontiers in Movement Science and Sport Psychology, a specialty of Frontiers in Psychology.

Copyright $\odot 2010$ Ong, Bowcock and Hodges. This is an open-access article subject to an exclusive license agreement between the authors and the Frontiers Research Foundation, which permits unrestricted use, distribution, and reproduction in any medium, provided the original authors and source are credited. 